Article

\title{
A Network-Based Method for the EMU Train High-Level Maintenance Planning Problem
}

\author{
Jianping Wu, Boliang Lin * (D), Jiaxi Wang and Siqi Liu \\ School of Traffic and Transportation, Beijing Jiaotong University, Beijing 100044, China; \\ 15114205@bjtu.edu.cn (J.W.); 13114236@bjtu.edu.cn (J.W.); 15114206@bjtu.edu.cn (S.L.) \\ * Correspondence: bllin@bjtu.edu.cn; Tel.: +86-108-516-7149
}

Received: 9 November 2017; Accepted: 19 December 2017; Published: 21 December 2017

\begin{abstract}
Electric Multiple Unit (EMU) high-level maintenance planning is a typical discrete system. EMU high-level maintenance (HM) planning determines when to undergo HM or execute transportation task for train-sets, based on practical requirements such as passenger transport demand, workshop maintenance capacity, and maintenance regulations. This research constructs a time-state network that can display the transformation processes between different states. On this basis, a path based model and its improvement are developed to minimize the HM costs with consideration of all necessary regulations and practical constraints. To handle the solution space, a path set generation method is presented. A real-world instance from Shanghai Railway, which is the largest affiliate in China Railway Corporation, was conducted to demonstrate the efficiency and effectiveness of the proposed approach, which indicates that the model can be solved to optimum within short computational times by the state-of-the-art solver Gurobi. Moreover, a sensitivity analysis was also performed to evaluate the effects of the variation in average daily operating mileage, HM capacity at the depot and the assumed minimum value of cumulative mileage.
\end{abstract}

Keywords: EMU train; high-level maintenance; time-state network; path set

\section{Introduction}

According to the statistical bulletin of National Railway Administration of the People's Republic of China, the railway passenger traffic volume reached 357 million during the Spring Festival Travel Rush in 2017 (from 13 January to 21 February, 40 days in total), among which people travelling by high-speed rail accounts for $51.4 \%$. High-speed railway operated by China Railway Corporation $(\mathrm{CR})$ is more and more popular for its convenience and comfortableness. How to supply enough rolling stock to fulfill heavy transportation tasks in tourist rush season (Spring Festival Travel Rush in particular) has long been the difficult problem for CR. The high purchase cost and complicated maintenance system of electric multiple unit (EMU) train-sets, which is the unique vehicle running on China high speed railway, makes the problem even worse.

In the CR system, a regular preventive maintenance mechanism is implemented for EMU train-sets. There are five levels of maintenance, e.g., the first-level (daily inspection), second-level (special inspection), third-level (bogie inspection), fourth-level (system decomposition inspection) and fifth-level (general inspection). Maintenances at the first and second level are both operational inspections with short inspection cycles and inspection service time. Typically, the maintenance plans of these two levels are co-optimization with the rolling stock assignment plan. The rest levels belong to high-level maintenance (HM). The high-level maintenance plan (HMP) must be considered in advance by the management (see Figure 1) because it requires a long time for the maintenance procedures, and the workshop has limited capacity, and it is scheduled once a year. It is an important objective pursued by a railway operator to supply enough available EMU train-sets for passenger transport demand and to reduce the major maintenance costs. 


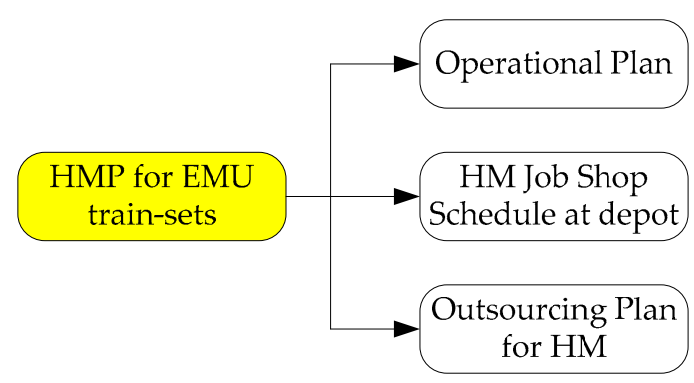

Figure 1. The high-level maintenance plan (HMP) process at China Railway Corporation (CR). EMU: electric multiple unit; HM: high-level maintenance.

HMP aims to make a tactical plan to balance maintenance work under the limited capacity with a long horizon, and try to avoid concentrated maintenances on travel peak periods. Because the process of $\mathrm{HM}$ is time-consuming, it will lead to the lack of train-sets for undertaking transportation tasks when lots of them be sent to workshop in tourist rush season. However, the start time for HM can be adjusted during the given interval according to the maintenance regulations. Therefore, this paper focuses on the optimization of HMP, which is of great significance in supply enough train-set for passenger transport peak demand. In the meantime, it also provides a decision support for the capacity configuration of the workshop.

For the maintenance system, Stuchly et al. [1] analyzed the maintenance management systems, and developed a maintenance management system based on reliability-centered maintenance which could help engineers to schedule the maintenance scheme reasonably according to the technical conditions reflected by the EMU real-time monitoring data. Shimada [2] introduced a new maintenance system of accident prevention based on the maintenance technology situation of Japanese Shinkansen, thereby reducing the maintenance costs and improving the utilization efficiency of EMU. Cheng and Tsao [3] proposed a selection strategy of EMU maintenance based on the characteristics of the preventive and corrective maintenances. The estimation method for the spare parts' quantities and replacement intervals of specific components of EMU were also provided.

For the optimization of EMUs maintenance plan, the existing literature is more concerned about the first- and the second-level maintenances. Maróti and Kroon [4,5] developed a multicommodity flow model for preventive maintenance routing. Given that the EMU requires maintenance in the forthcoming one to three days, the operation schedule must be adjusted so that these urgent units reach the maintenance facility in time. Rezvanizaniani et al. [6] discussed the implementation of Reliability Centered Maintenance to make rolling stock maintenance of the Raja Passenger Train Corporation more cost effective by reducing erroneous maintenance and unnecessary maintenance. Tsuji et al. [7] analyzed the influence factors of EMU operation and maintenance problems combined with the Japanese Shinkansen, and developed a novel approach based on ant colony optimization to solve the problems. Wang et al. [8] proposed a 0-1 programming model to study EMU maintenance, but the model cannot be applied in high-level maintenance practice. Giacco et al. [9] provided a mixed-integer linear-programming formulation for integrating short-term maintenance planning in a network-wide railway rolling stock circulation problem, and the optimization objective is to minimize the cost with service pairings, empty runs, and short-term maintenance tasks. Lai et al. [10] studied an exact optimization model to improve the efficiency in rolling stock usage with consideration of practical operation constraints and multi-level maintenance. Compared to the manual process, a hybrid heuristic process is developed to improve solution quality and efficiency. In the field of EMU high-level maintenance research, Sriskandarajah et al. [11] optimized the EMU overhaul plan using the genetic algorithm. The genetic algorithm improves the quality of plan and reduces the costs of operation. Lin et al. [12] proposed a non-linear 0-1 programming model, and a state function was established to describe whether a train is under maintenance. Li et al. [13] developed a method to forecast the maintenance quantity of EMU in an arbitrary time period, accurately and efficiently. 
Similar to EMU maintenance scheduling, scheduled maintenance planning problems have been studied in other fields; Ziarati et al. [14] studied the locomotive operation by constructing a large integer programming model, and they focused on the influence of maintenance for operation. Lingaya et al. [15] described a model for operational management of locomotive hauled railway cars. They sought for a maximum expected profit schedule that satisfied various constraints, among them maintenance requirements were also included. Wang et al. [16] developed a 0-1 integer programming model to study locomotive operation and maintenance, and applied genetic algorithm to solve the model. However the model ignored the maintenance capacity. Moudania and Félix $[17,18]$ studied the problems of assigning planes to flights and of fleet maintenance operations scheduling. And they also proposed a dynamic approach to cope with the fleet assignment problem and a heuristic technique to solve the embedded maintenance scheduling problem. Budai et al. [18] presented a mathematical formulation for the long-term planning of railway maintenance works. The objective is to minimize the time required for maintenance, expressed as a cost function. Heuristic algorithms computed nearly optimal solutions by combining maintenance activities on each track. Mehmet and Bilge [19] developed integer linear programming model by modifying the connection network representation which provided feasible maintenance routes for each aircraft in the fleet over a weekly planning horizon, with the objective of maximizing the utilization of the total remaining flying time of fleet. The proposed model is solved by using branch-and-bound under different priority settings for variables to branch on. In addition, Grigoriev et al. [20] studied the problem of scheduling maintenance services, and the objective was to find a cyclic maintenance plan of a given length that minimized total operation costs. A branch and price algorithm was applied to solve the model. Lv et al. [21] proposed a novel method to solve the shop scheduling problem featured with uncertain processing times. Shan et al. [22] utilized the Markov property and neural network ensemble to construct an estimated matrix that combines the interaction of the different local factors.

Compared to the existing researches, this research includes some highlights as follows. (1) A 0-1 integer programming model and its improvements were proposed to reduce the costs of $\mathrm{HM}$ with consideration of all necessary regulations and practical constraints, especially for the passenger transport peak demand; (2) Taking into account the practice constraints, we constructed a time-state network for optimizing HMP; (3) the generation method of the path set was presented to solve the mathematical model effectively and efficiently by controlling the solution space.

This paper is organized as follows. Section 2 aims to systematically describe the HMP problem in the CR system. Section 3 constructs a time-state network for optimizing the HMP, then an integer programming model and its improvements were proposed to minimize the HM costs, followed by the path set generation method to solve the mathematical models effectively and efficiently in Section 4. An empirical case and sensitivity analysis using standard optimization solvers are presented in Section 5.

\section{HMP Problem at CR}

The HMP is a plan that determines when to undergo HM or execute transportation task for train-sets. A quality plan should supply enough available train-sets for passenger transport demand, satisfy maintenance capacity constraints, comply with the maintenance system and reduce the maintenance cost as much as possible. In other words, this process has to follow a set of constraints, including (1) demand, and (2) maintenance constraints. In the CR system, an EMU train-set usually consists of eight or sixteen units of power and non-power rolling stock permanently connected together, which marked 8-cars or 16-cars. Therefore, each unit within a train-set operates daily tasks and undergoes inspections together. We regarded a train-set that included eight cars or 16 cars as a nature set, and regarded 8 cars as a standard set, then the train-set including 16-cars equaled to two standard sets. 


\subsection{Demand}

In CR, the travel demand fluctuates over different periods. There are several peak demands in one year, and some of them last for one to two months, e.g., Spring Festival and Summer Holiday, others for several days, such as New Year's Day, Tomb-sweeping Day, Labor Day, Dragon Boat Festival, Mid-autumn Day, and National Day. Aimed at these peak demands, CR has drawn passenger train diagram based on relevant documents and files, and all subsidiaries have drawn up corresponding rolling stock scheduling. The HMP is to ensure that there is supply of enough well-conditioned train-sets to implement the rolling stock scheduling (travel rush in particular).

\subsection{Maintenance}

The maintenance requirement sets the limit on how much distance and time a train-set can operate before the next mandatory maintenance. Table 1 shows the HM requirement for the "CRH1" series EMU train-set in terms of cumulative operating mileage and cumulative operating days.

Table 1. Maintenance regulations for CRH1 series in China Railway.

\begin{tabular}{cccc}
\hline Type & Third-Level & Fourth-Level & Fifth-Level \\
\hline CRH1B & $(120 \pm 10) \times 10^{4} \mathrm{~km}$ & $(240 \pm 10) \times 10^{4} \mathrm{~km}$ & $(480 \pm 10) \times 10^{4} \mathrm{~km}$ \\
CRH1E & or 3 years & or 6 years & or 12 years \\
\hline
\end{tabular}

For example, a train-set, CRH1B, should undergo the third-level maintenance after operating for $1,200,000 \mathrm{~km}$ or for three years before it can be assigned to the next operation utilization path with actual train trips. Furthermore, the HM requirement has a floating range. For example, cumulative operating mileage of the third-level maintenance for CRH1B is [1,100,000 km, 1,300,000 km], i.e., a CRH1B train-set will undergoes the procedures of third-level maintenance when its accumulated operating mileage is greater than 1,100,000 km and less than 1,300,000 km from the last HM.

As seen in Table 1, the threshold of fourth-level and fifth-level are twice and four times, respectively, greater than the threshold of third-level. The interval between two consecutive HMs should be less than the third-level requirements according to CR [23]. Therefore, we can draw the conclusion that the HM requirements are equal to third-level requirements, and the train-set undergoes two consecutive HMs at different levels accordingly. On the basis of this rule, the HM cyclic graph could be drawn, as shown in Figure 2. Based on the sequence of HM cyclic graph and the HM requirement, the train-set accepts the different levels of HMs in turn until they are scrapped. Moreover, the maintenance procedures of higher-level maintenance include all maintenance procedures in lower-level maintenance. Thus, after one class of maintenance process, all cumulative operating mileage and days associated with that class and the corresponding lower-level classes of maintenance are all set to zero. Such as, all associated cumulative operating mileage and days for fourth-level and third-level should be set to zero after fourth-level.

According to the $\mathrm{CR}$, the HM requirements depends on the cumulative operating mileage mainly and the cumulative operating days as a supplement, and whichever comes first. The average daily operating mileage of CRH1B and CRH1E is $1600 \mathrm{~km}$ and $1500 \mathrm{~km}$ respectively. As the simple mathematical calculation based on the data in Table 1, we can conclude that the cumulative operating mileage for any train-set will fulfill firstly, such as a CRH1E EMU train-set, $(1,200,000 \mathrm{~km}) /(1500 \mathrm{~km} /$ day $)=800$ days $<3$ years. Therefore, we could only take into account the cumulative operating mileage requirement and ignore the cumulative operating days in this paper. 


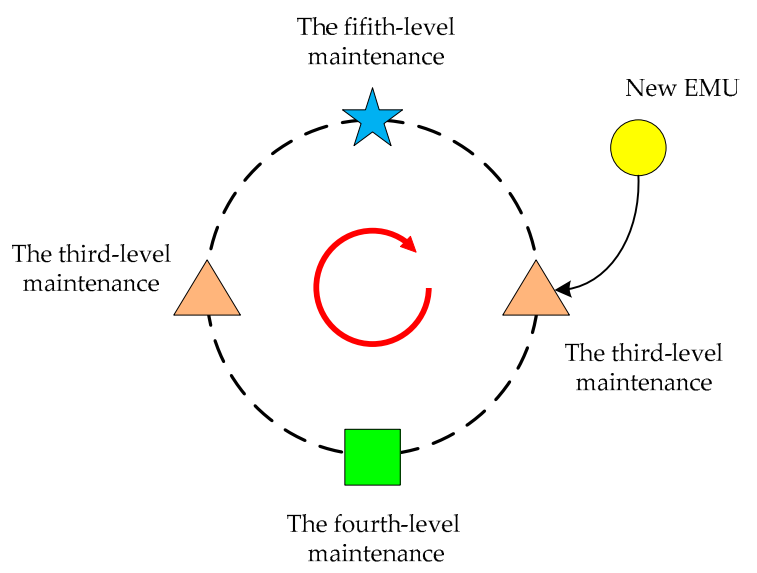

Figure 2. The HM cyclic graph.

The average daily operating mileage is an estimated value based on historical data to calculate the eta (estimated time of arrival) of HM. This is a reasonable assumption, because at the operational level, we can assign itineraries to train-sets on each day so as to ensure that the true average number of operating mileage per day is close to the average daily operating mileage during the long planning horizon.

When the train-set needed HM, it would be sent to a workshop. In order to keep away from the peak season of travel, some train-sets may choose to undergo the HM before the rush, and others may conduct maintenance after the rush. However, too much postponing will be bad for operation safety, and too much time ahead of the estimated time of arrival (ETA) will waste the operating mileage. In addition, as the HM is costly, frequent maintenance will lead to a great waste of resources. Therefore, under the premise of safety operation, the start time of HM should be postponed as soon as possible.

Aside from these requirements, every depot and plant has a certain simultaneous maintenance capacity and receiving capacity that can be performed per day. Meanwhile, when the train-set was sent to a workshop, there exists an interval for receiving the next train-set according to the capacity of workshop and the size of the train-set (8-cars or 16-cars).

\section{Methodology}

In this section, a time-state network, a corresponding mathematical model and its improvements are presented. We divided the state of the train-set into four states, i.e., the available state, third-level maintenance state, the fourth-level maintenance state, and the fifth-level maintenance state. There exists no waiting state, it is because of the start time that HM can be adjusted flexibly during the given interval. The available state includes the operation state, the first-level maintenance state and the second-level maintenance state. But it was unnecessary to concern the rolling stock assignment and low-level maintenance plan in the long planning horizon for HMP, and the average daily operating mileage based on the historical data was valid to describe the available state.

\subsection{Time-State Network}

A time-state network is designed based on graph theory to analyze HMP. In China high speed railway, train-sets operate the timetable during the daytime and get rest or lower level maintenance during the night. Furthermore, the working time of the maintenance workshop is also during the daytime. Therefore, we constructed a time-state network by setting the night as a node, and regarded the duration of daytime as an arc that connects adjacent nodes (see Figure 3). The horizontal direction represents the elapse of time, and the vertical direction shows different states of train-sets. 
Directed graph $G$ consists of the node set $V$ and the arc set $A, G=(V, A)$. The last column denotes virtual hyper-nodes in the planning horizon. The notations, including the indices, parameters, and sets, are listed in Table 2.

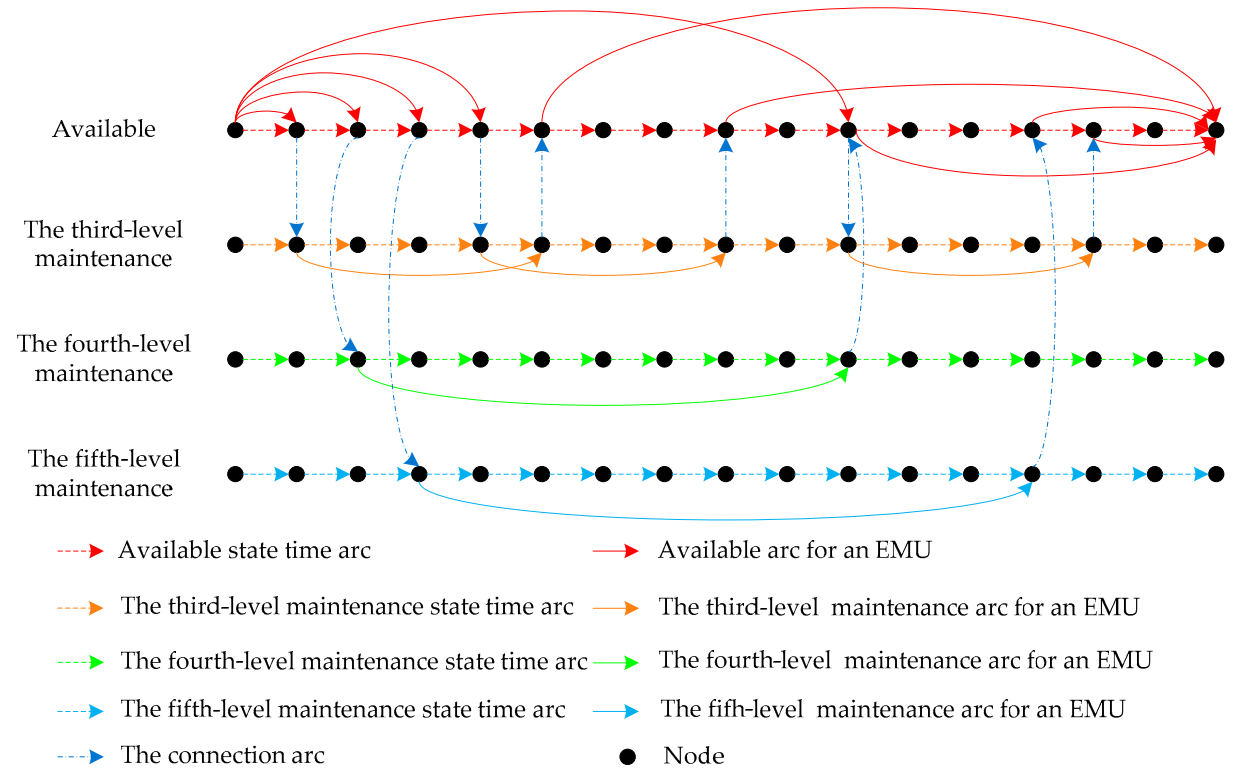

Figure 3. Schematic diagram of connection relationship between available and HM states for EMU.

Table 2. Notation of indices, sets, and parameters.

\begin{tabular}{cl}
\hline Notation & \\
\hline Indices & \\
\hline$i, j$ & The state of the train-set: $i, j=1,2,3,4$, denote available state, the third-, fourth- \\
$k, h$ & and fifth-level maintenance state, respectively \\
$e$ & The ordinal number of the column (day): $k, h=1,2,3, \ldots, T$ \\
\hline Parameters & \\
\hline$v_{i k}$ & The train-set: $e \in E$ \\
$a$ & The arc: $a \in A$ \\
$a_{i k}$ & The time arc following the node $v_{i k}: a_{i k} \in A_{i}$ \\
$a_{i j}^{k}$ & The connection arc from $v_{i k}$ to $v_{j k}: i \neq j$ \\
$a_{i}^{h k}$ & The available/maintenance arc from $v_{i h}$ to $v_{i k}$ \\
$w_{i k}$ & The volume value of time arc \\
$b_{1}\left(a_{1, k}\right)$ & The minimum value of $w_{i k}$ for arc $a_{1, k}$ \\
$b_{i}$ & The maximum value of $w_{i k}$ for arc $a_{i k}: i=2,3,4$ \\
$T$ & The length of planning horizon \\
$r$ & The path on the time-state network: $r \in R$ \\
$t$ & The time point from the available state converted to the maintenance state \\
$g$ & The level of maintenance \\
$\mu$ & The length of maintenance service time \\
\hline Sets & \\
\hline & \\
\hline & Set of train-sets \\
$V$ & Set of all arcs \\
$A_{i}$ & Set of all nodes \\
$R$ & Set of time arcs: $A_{i} \subset A, i=1,2,3,4$ \\
\hline
\end{tabular}

A dashed line presents the time arc $a_{i k}$ in Figure 3. There are four kinds of time arcs that exist in the time-state network (distinguished by different color), which represent the available state, and third-level, fourth-level and fifth-level maintenance state, respectively. Then, we explain the 
weight of these time arcs as follows. Let $w_{i k}$ denote the number of train-sets on the $k$-th day and in the $i$-th state. To successfully undertake the transportation tasks on the $k$-th day, the $w_{1, k}$ must greater than or equal to $b_{1}\left(a_{1, k}\right)$, i.e., $w_{1, k} \geq b_{1}\left(a_{1, k}\right)$. Note that the value of $b_{1}\left(a_{1, k}\right)$ is the least demand for available states, and it is variable according to travel demand on the $k$-th day. Let $w_{2, k}$ denote the number of train-sets in third-level maintenance state on the $k$-th day. Due to the limited service capacity of the workshop, $w_{2, k}$ has its maximum value $b_{2}$. Similarly, $w_{3, k} \leq b_{3}$ and $w_{4, k} \leq b_{4}$. Note that $b_{2}, b_{3}, b_{4}$ is fixed, but $b_{1}\left(a_{1, k}\right)$ is variable.

The process of train-sets entering or leaving workshop are relatively stochastic in practical, so we did not take time consumption into consideration for this. It was assumed that the train-set can switch to maintenance state on the same day after operation ending, and vice versa, which is depicted by a deep-blue dash-dotted arc in Figure 3. The direction of the arc, denoted by $a_{i j}^{k}$, signifies the state transition direction. In addition, $a_{1, k}$ and $a_{2, k}, a_{1, k}$ and $a_{3, k}, a_{1, k}$ and $a_{4, k}$ can be linked together, respectively, by $a_{i j}^{k}$. However, it cannot be connected between the $a_{2, k}, a_{3, k}$ and $a_{4, k}$. In other words, the state of a train-set could transform from available to maintenance, or from maintenance to available, but cannot transform from a level of maintenance to another level.

HMP determines on which day the train-set should go to workshop and which level of maintenance the train-set should undertake. On the network, this process can be shown as a path by connecting different arcs throughout the whole planning horizon. Such as the path $r_{0}$ (see in Figure 4):

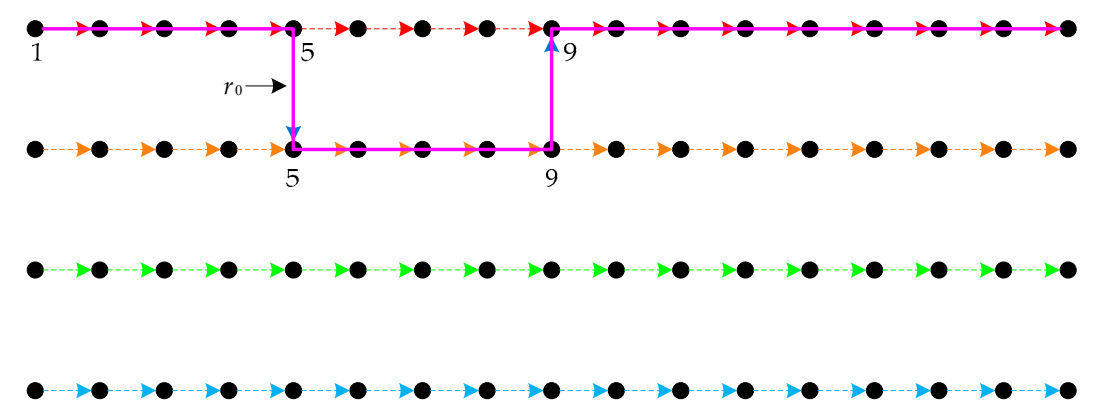

Figure 4. Path $r_{0}$.

As shown in Figure 4, path $r_{0}$ describes that a particular train-set is in an available state from the first day to the fourth day; then undergoes third-level maintenance according to maintenance requirements from the fifth day to the eighth day; after that, this train-set goes back to undertake transport tasks from the ninth day to the end of planning horizon. In this way, all the state and state transition processes for a train-set in the planning horizon are depicted by a path, which is decided by the time point from the available state transform to the maintenance state, the level of maintenance, and the length of maintenance service time. For any particular train-set, the level of maintenance and the length of maintenance service time are known in advance, and the key decision is the time point from the available state transform to the maintenance state. Therefore, the HMP problem could be converted into a pathway chosen problem on the time-state network. We optimized the latter to realize the optimization of the former in this paper.

In addition, in order to show the specific state for a train-set intuitively on the network, we connect the time arcs in the same state to form an arc chain denoted by $a_{i}^{h k}$, which is depicted by a solid line and painted with the homologous color. At the end of a planning horizon, let all the solid line arcs be directed to the hyper-node in each row. In Figure 3, a train-set is kept in an available state from $v_{1,1}$ to $v_{1,5}$, which can be depicted by a red solid line from $v_{1,1}$ to $v_{1,5}$. The length of this red line denotes the duration of available state for train-set. Similarly, this train-set is kept in third-level maintenance state from $v_{2,5}$ to $v_{2,9}$, which can be denoted by an orange solid line from $v_{2,5}$ to $v_{2,9}$. The length of this line represents the third-level maintenance service time. It should be pointed out that the Figure 3 is only a sketch map, whose time span do not presents the actual time. 


\subsection{Mathematical Model}

Based on the time-state network, a mathematical model is proposed that inspired by classic arc-path model. The decision variables are defined as follows:

$$
x_{e}^{r}=\left\{\begin{array}{cc}
1 & \text { EMU } e \text { chooses the path } r, e \in E, r \in R \\
0 & \text { otherwise }
\end{array}\right.
$$

And define the associated parameter is as follows:

$$
\delta_{r}^{a}=\left\{\begin{array}{cc}
1 & \text { Arc } a \text { in the path } r, r \in R, a \in A \\
0 & \text { otherwise }
\end{array}\right.
$$

Associated parameter $\delta_{r}^{a}$ denoted by a matrix, describes the inclusion relation between arc and path. So, $w_{i k}$ can be describes as follows:

$$
w_{i k}=\sum_{r \in R} \sum_{e \in E} x_{e}^{r} \delta_{r}^{a_{i k}} \quad \forall a_{i k} \in A_{i}, i=1,2,3,4, k=1,2, \ldots, T
$$

\begin{tabular}{|c|c|}
\hline Parameters & Description \\
\hline$L_{e}^{\text {upper }}$ & $\begin{array}{l}\text { The upper bound of the allowable cumulative mileage before } \mathrm{HM} \text { according } \\
\text { to the maintenance requirement. }\end{array}$ \\
\hline$L_{e}^{\text {lower }}$ & $\begin{array}{l}\text { The lower bound of the allowable cumulative mileage before } \mathrm{HM} \text { according } \\
\text { to the maintenance requirement. }\end{array}$ \\
\hline$L_{e}^{\min }$ & $\begin{array}{l}\text { The assumed minimum value of cumulative mileage before } \mathrm{HM} \text { for } \\
\text { convenient calculation. }\end{array}$ \\
\hline$L_{e}^{i d e a l}$ & $\begin{array}{l}\text { The ideal cumulative mileage before } \mathrm{HM} \text { according to the maintenance } \\
\text { requirement. }\end{array}$ \\
\hline$l_{e}$ & $\begin{array}{l}\text { The state variable that indicates the expected operating mileage since the last } \\
\text { HM when } e \text { was sent to workshop. }\end{array}$ \\
\hline$\overline{l_{e}}$ & The average daily operating mileage for $e$ that equals to its types. \\
\hline$l_{e}^{0}$ & $\begin{array}{l}\text { The accumulative operating mileage since last } \mathrm{HM} \text { for } e \text { at the beginning of } \\
\text { planning horizon. }\end{array}$ \\
\hline$t_{e}^{0}$ & $\begin{array}{l}\text { The accumulative operating days since last } \mathrm{HM} \text { for } e \text { at the beginning of the } \\
\text { planning horizon. }\end{array}$ \\
\hline$\alpha$ & $\begin{array}{l}\text { The size of train-set, if the train-set includes eight cars, then } \alpha=1 \text {; otherwise, } \\
\alpha=2 \text {. }\end{array}$ \\
\hline
\end{tabular}

Another set of parameters in the model are listed in Table 3.

Table 3. Another set of parameters in the model. HM: high-level maintenance.

The objective function of the mathematical model is to minimize the costs of HM. We did not consider the optimization of the HM processes, and regarded it as a fixed value in this paper. So, the objective function could be described by decreasing HM frequency. The practical method of decreasing frequency is to increase the interval of two consecutive HMs, i.e., increasing the actual operating mileage between two consecutive HMs. Moreover, according to the maintenance requirements, the actual operating mileage since the last HM cannot exceed the upper limit of the floating range. Therefore, the objective function could be described by minimizing the D-value between 
the maximum cumulative operating mileage of maintenance requirements and the expected operating mileage for all EMUs. As mentioned above, the objective function can be simply described as follows:

$$
\min \mathrm{W}=\sum_{e \in E} L_{e}^{\text {upper }}-l_{e}
$$

If a train-set is undergoing inspection at the beginning of the planning horizon, $l_{e}^{0}$ and $t_{e}^{0}$ will take a negative value for simplifying the model in this paper, and $t_{e}^{0}$ presents that $\left|t_{e}^{0}\right|$ days are remaining to finish the inspection at this moment. So, we have the following formula:

$$
l_{e}^{0}= \begin{cases}l_{e}^{0} & t_{e}^{0} \geq 0 \\ t_{e}^{0} \overline{l_{e}} & t_{e}^{0}<0\end{cases}
$$

Then, $l_{e}$ can be calculated as the following equation:

$$
l_{e}=l_{e}^{0}+(k-1) \cdot \overline{l_{e}} \sum_{r \in R} x_{e}^{r} \delta_{r}^{a_{1 j}^{k}} \quad \forall e \in E, j=2,3,4, k=1,2, \ldots, T
$$

Constraints of the model are mainly derived from the following several aspects.

(1) For safety reasons, the expected operating mileage since last HM have to be less than or equal to the maximum value of cumulative operating mileage, according to HM requirements. On the other hand, the expected operating mileage should be greater than or equal to the minimum value. To search the feasible solution effectively and to control the solution space, we have the assumed minimum value instead of the minimum value from $\mathrm{HM}$ requirements, i.e.,

$$
L_{e}^{\text {min }} \leq l_{e} \leq L_{e}^{\text {upper }} \forall e \in E
$$

(2) According to the proposed approach, any $e$ can choose one and only one path on the time-state network, which is the uniqueness constraint of the path. Its formulation is listed as follows:

$$
\sum_{r \in R} x_{e}^{r}=1 \quad \forall e \in E
$$

(3) The minimum number of train-sets in available state is variable according to travel demand, and it should be guaranteed. To describe this requirement, a set of constraints established as follows:

$$
\sum_{r \in R} \sum_{e \in E} x_{e}^{r} \delta_{r}^{a_{1, k}} \alpha_{e} \geq b_{1}\left(a_{1, k}\right) \quad \forall a_{1, k} \in A_{1}, k=1,2, \ldots, T
$$

(4) The number of train-sets in any level of maintenance state should not exceed the capacity of simultaneously maintenance service. Similarly, a set of constraints established as follows:

$$
\sum_{r \in R} \sum_{e \in E} x_{e}^{r} \delta_{r}^{a_{i k}} \alpha_{e} \leq b_{i} \quad \forall a_{i k} \in A_{i}, i=2,3,4, k=1,2, \ldots, T
$$

(5) Only a certain number of train-sets can be accepted in the workshop over several days, which is due to the limited maintenance resources in some workstations. An associated parameter $\theta_{r}^{a}$ is introduced for conveniently describing this relationship. The definition of $\theta_{r}^{a}$ is as follows:

$$
\theta_{r}^{a}=\left\{\begin{array}{cc}
1 & \text { Arc } a \text { in the path } r, r \in R, a \in A \\
0 & \text { otherwise }
\end{array}\right.
$$


The arc $a$ in $\theta_{r}^{a}$ can be regarded as the first few days of the maintenance process, and the duration is equal to the interval for the receiving train-set. The associated parameter $\theta_{r}^{a}$ can be calculated beforehand, and its meaning can be visually represented, as shown in Figure 5.

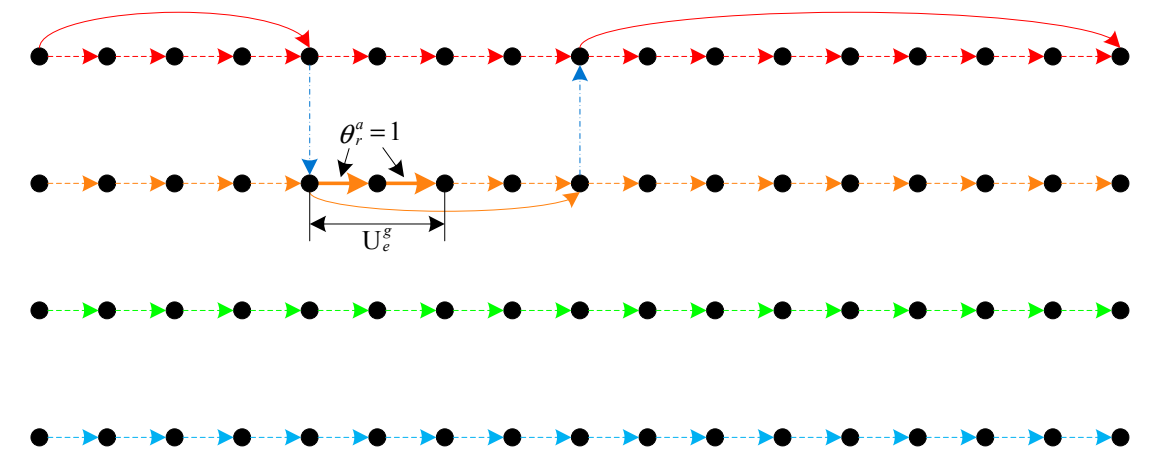

Figure 5. The interval of accepting train-sets for workshop.

$\mathrm{U}_{e}^{i}(i=2,3,4)$ is the duration of the minimum interval for receiving the another train-set after train-set $e$ be received in the $i$-th state, e.g., $\mathrm{U}_{15}^{2}=2$ describes a train-set (No. 15) entering into the workshop for undergoing third-level maintenance, and the next train-set could be sent to the workshop at two or more days later.

Therefore, the constraints of receiving train-sets in the workshop can be presented as follows:

$$
\sum_{r \in R} \sum_{e \in E} x_{e}^{r} \theta_{r}^{a_{i k}} \leq N_{i} \quad \forall a_{i k} \in A_{i}, i=2,3,4, k=1,2, \ldots, T
$$

where $N_{i}$ is the number of acceptable train-sets at the same time in $i$-th state.

(6) Although each train-set can choose one path in the path set $R$, the choice is not discretionary. The train-set can choose the path that involves corresponding information of maintenance level and maintenance service time. To this end, we introduced a parameter $\lambda_{e}^{r}$, which describes a consistent one-to-one match between each train-set and each path, as follows:

$$
\lambda_{e}^{r}=\left\{\begin{array}{cc}
1 & \text { path } r \text { could be chosen by } e, e \in E, r \in R \\
0 & \text { otherwise }
\end{array}\right.
$$

The value of $\lambda_{e}^{r}$ should be calculated beforehand. Then, the valid inequality constraints are established:

$$
x_{e}^{r} \leq \lambda_{e}^{r} \quad \forall e \in E, r \in R
$$

From the above analysis, the model is summarized as follows.

Model I:

$$
\min Z=\sum_{e \in E} L_{e}^{\text {upper }}-l_{e}
$$

s.t. constraints (4)-constraints (10) and:

$$
x_{e}^{r} \in\{0,1\} \quad \forall e \in E, r \in R
$$

\subsection{Model Improvements}

There are very limited selections to choose a reasonable path from the path set $R$ for a particular train-set, according to the characteristic of HMP problem. For instance, a train-set should have undergone third-level maintenance during the planning horizon. Then, the path involving the fourth-level or fifth-level maintenance state time arc cannot be chosen. Moreover, according to the constraints (5), just part of the paths involved the third-level maintenance state time arc can be 
selected. Constraints (10) can effectively reduce the solution space, but there still exists a lot of invalid computation when solving the model I. Therefore, we can generate a path subset for each train-set respectively, thereby improving the speed of the solving model. Although it is probable that there exist identical paths in two or more path sets, it is convenient to solve the model. Based on the analysis above, the model I can be improved as follows:

Model II:

$$
\min Z=\sum_{e \in E} L_{e}^{\text {upper }}-l_{e}
$$

s.t. Constrains (5) and:

$$
\begin{gathered}
l_{e}=l_{e}^{0}+(k-1) \cdot \bar{l}_{e} \sum_{r \in R_{e}} x_{e}^{r} \delta_{e}^{r a_{1 j}^{k}} \quad \forall e \in E, j=2,3,4, k=1,2, \ldots, T . \\
\sum_{r \in R_{e}} x_{e}^{r}=1 \quad \forall e \in E \\
\sum_{r \in R_{e}} \sum_{e \in E} x_{e}^{r} \delta_{r}^{a_{1, k}} \alpha_{e} \geq b_{1}\left(a_{1, k}\right) \quad \forall a_{1, k} \in A_{1}, k=1,2, \ldots, T \\
\sum_{r \in R_{e}} \sum_{e \in E} x_{e}^{r} \delta_{r}^{a_{i k}} \alpha_{e} \leq b_{i} \quad \forall a_{i k} \in A_{i}, i=2,3,4, k=1,2, \ldots, T \\
\sum_{r \in R_{e}} \sum_{e \in E} x_{e}^{r} \theta_{r}^{a_{i k}} \leq N_{i} \quad \forall a_{i k} \in A_{i}, i=2,3,4, k=1,2, \ldots, T . \\
x_{e}^{r} \in\{0,1\} \quad \forall e \in E, r \in R_{e}
\end{gathered}
$$

\section{Path Set Generation}

To solve the model above, we must generate $R_{e}$ for each $e(e \in E)$ on the network. In order to achieve this purpose, the subsequent arc set followed each arc should be determined in advance. In this section, the subsequent arc set followed each arc was given firstly, then the specific generation steps of path set for each train-set were proposed.

\subsection{Subsequent Arc Sets}

According to the network design, the path is an arc chain that formed by a variety of end-to-end arcs throughout the whole planning horizon. To find out these arc chains, we should recognize the subsequent arc set of each type of arc first. At the beginning of a planning horizon, the train-set may be in available or in a maintenance state.

(1) The subsequent arc set of $a_{1 k}$ is described as follows: As for the train-set that is available, the subsequent state of it may be still be kept available or turned to the HM state. So, the subsequent arc set of $a_{1 k}$ is $\left\{a_{1, k+1}, a_{1 j}^{k+1}(j=2,3,4)\right\}$.

(2) To understand the definition of the subsequent arc set of $a_{1 j}^{k}(j=2,3,4)$, we can consider this case: Along with the train-set going to the workshop, the maintenance procedures of a certain level will be conducted on it. Thus, the subsequent arc set of $a_{1 j}^{k}(j=2,3,4)$ is $\left.\left\{a_{j k} \mid j=2,3,4\right)\right\}$.

(3) The subsequent arc set of $a_{j k}(j=2,3,4)$ is illustrated as follows: When a certain level of HM for train-set is completed, the state of the train-set will transform to being available; but, if further maintenance is needed, the subsequent arc should be $a_{j, k+1}(j=2,3,4)$. Therefore, $\left\{a_{j 1}^{k+1}\right.$, $\left.a_{j, k+1} \mid j=2,3,4\right\}$ is the subsequent arc set of $a_{j k}(j=2,3,4)$.

(4) The subsequent arc set of $a_{j 1}^{k}(j=2,3,4)$ is defined as follows: Once the HM of the train-set is completed, the state of train-set will transform to being available. So the subsequent arc set is $\left\{a_{1 k}\right\}$. 


\subsection{Steps for Path Set Generation}

After clearing the subsequent arc set for each arc on the network, the path set $R_{e}$ for each $e$ can be generated. The processes of path generation depends on time based depth-first, and the specific steps are as follows:

Step 1: $G^{\prime}=G$;

Step 2: According to the initial state of train-set $e$, we assign the initial arc and clear the arc's type on the network $G^{\prime}$;

Step 3: Based on the connection sequence of arcs mentioned at Section 4.1, the time based depth-first search algorithm is applied to find the subsequent arc considering constraints (5) on the network $G^{\prime}$. If no subsequent arc is found, then turn to Step 6. Otherwise, turn to Step 4;

Step 4: Judge whether or not the subsequent arc reaches at the virtual hyper-node. If yes, turn to Step 5; otherwise, turn to Step 3;

Step 5: Connect all arcs in the order and output the path $r$. Add $r$ into $R_{e}$, and delete the $a_{1 j}^{k}$ $(j=2,3,4)$ existing in path $r$. Then reconstruct the network $G^{\prime}$ and go back to Step 2;

Step 6: End the path generation processes and output $R_{e}$.

Reapply the algorithm above and generate $R_{e}$ on the network for each $e(e \in E)$.

Then, we can also get the universal set $R$ of all train-sets for model I as follows:

$$
R=\underset{e \in E}{\cup} R_{e}
$$

Meanwhile, $\lambda_{e}^{r}$ can be assigned by Equation (18).

Based on the method of path set generation, the constraints (5) in model I and model II can be removed.

\section{Empirical Case Study and Sensitivity Analysis}

In this section, we implement the proposed method at Shanghai Railway, which owns the most EMU train-sets in the CR system. An empirical case study is selected and analyzed to test the computational efficiency and effectiveness of the proposed integer linear programming (ILP) models, including model I and model II. Finally, the sensitivity analysis is conducted to evaluate the effects of the variation in average daily operating mileage, HM capacity at the depot and the assumed minimum value of cumulative mileage. The proposed ILP models are solved by Gurobi; and all the computational experiments are coded in Python 3.6 and implemented within Spyder on a computer with a $3.2 \mathrm{GHz}$ processor and 4 GB RAM.

\subsection{Empirical Case Study}

Shanghai railway owns 91 CRH1 series and CRH380D EMU train-sets, which including 24 sets of CRH1B (16-cars), 19 sets of CRH1E (8-cars) and 48 sets of CRH380D (8-cars). Among these trains, 11 sets of CRH1B, eight sets of CRH1E, and 41 sets of CRH380D needed maintaining during the planning horizon. The start date of the planning horizon is 18 August 2016, denoted by number 1 , and the end date is 1 February 2018, so the length of the planning horizon is 533 days, i.e., $T=533$. The maintenance regulations for EMU train-sets are listed in Table 1. Table 4 shows the initial condition of train-sets needed maintaining at the beginning of the planning horizon, including the ID, average daily operating mileage, type, initial cumulative mileage, maintenance level, maintenance level service time, the interval for delivering train-sets to the workshop, and the ETA and the deadline for HM. The eta of maintenance is calculated by the formula as follows:

$$
\operatorname{eta}_{e}=\left[\left(L_{e}^{\text {ideal }}-l_{e}^{0}\right) / \overline{l_{e}}\right]+1
$$

Similarly,

$$
\text { deadline }_{e}=\left[\left(L_{e}^{\text {upper }}-l_{e}^{0}\right) / \overline{l_{e}}\right]+1
$$


In order to calculate conveniently in this empirical case, a simple formula should be followed:

$$
L_{e}^{\text {min }}=L_{e}^{\text {upper }}-p \overline{l_{e}}
$$

where $p$ is the modified parameter for guaranteeing the feasible solution. According to Table 1 , we set $p=80$ (see Section 5.2), approximately, after multiple trials. If $L_{e}^{\min }<0$, then $L_{e}^{\min }=0$. Moreover, if many of train-set needed maintenance at the same time, we can increase the value of $p$ to enlarge the solution space to disperse the start date of HM under the limited maintenance capacity.

During the planning horizon, there exists four long travel rushes, including two spring festival, one spring season and one summer holiday. All maintenance work should be finished at the 532th day. According to practice, $b_{1}\left(a_{1, k}\right)$ is valued as follows:

$$
b_{1}\left(a_{1, k}\right)=\left\{\begin{array}{c}
112 \text { if } k \in[149,189) \\
107 \text { if } k \in[189,318) \\
108 \text { if } k \in[318,380) \\
115 \text { if } k=533 \\
105 \text { otherwise }
\end{array}\right.
$$

The unit of measurement of $b_{1}\left(a_{1, k}\right)$ and $b_{i}(i=2,3,4)$ is a standard set (a train-set includes eight cars), and the 16-car train-set is equal to two standard sets. All of the train-sets undergo third-level maintenance at the depot, and undergo fourth- and fifth-level maintenance at the plant. The maximal number of standard sets that can be maintained simultaneously at the depot for the third-level is seven, i.e., $b_{2}=6$. The maintenance capacity of the plants is sufficient. We set the simultaneous maintenance capacity for fourth-level and fifth-level as large positive numbers, e.g., $b_{3}+b_{4}=20$. Meanwhile, the receiving capability per day for depot and plant are both limited, e.g., $N_{2}=1$ and $N_{3}+N_{4}=2$. The unit of measurement of $N_{i}(i=2,3,4)$ is the natural set.

\begin{tabular}{|c|c|c|c|c|c|c|c|}
\hline $\mathbf{e}$ & $\overline{l_{e}}(\mathbf{k m})$ & $l_{e}^{0}(\mathbf{k m})$ & g & $\mu$ (d) & Interval (d) & $e t a_{e}$ & deadline $_{e}$ \\
\hline 1 & 1600 & $1,018,147$ & 3 th & 55 & 2 & 114th & 177th \\
\hline 2 & 1600 & $1,018,021$ & 3 th & 55 & 2 & 114th & 177th \\
\hline 3 & 1600 & 977,308 & 3th & 55 & 2 & 140th & 202th \\
\hline 4 & 1600 & 922,184 & 3 th & 55 & 2 & 174th & 237 th \\
\hline 5 & 1600 & 889,720 & 3 th & 55 & 2 & 194th & 257th \\
\hline 6 & 1600 & 820,787 & 3th & 55 & 2 & 238th & 300th \\
\hline 7 & 1600 & 801,365 & 3 th & 55 & 2 & 250th & 312th \\
\hline 8 & 1600 & 682,002 & 4 th & 60 & 1 & 324th & $387 \mathrm{th}$ \\
\hline 9 & 1600 & 528,475 & 4 th & 60 & 1 & 420th & 483th \\
\hline 10 & 1600 & 514,802 & 4 th & 60 & 1 & 429th & 491th \\
\hline 11 & 1600 & 434,547 & 4 th & 60 & 1 & 479th & 541th \\
\hline 12 & 1600 & $1,017,601$ & 3 th & 50 & 2 & 122th & 189th \\
\hline 13 & 1600 & 889,229 & 3 th & 50 & 2 & 208th & 274th \\
\hline 14 & 1600 & 801,234 & 3 th & 50 & 2 & 266th & 333th \\
\hline 15 & 1600 & 744,037 & 3 th & 50 & 2 & 304th & 371th \\
\hline 16 & 1600 & 545,587 & 3 th & 50 & 2 & 437th & 503th \\
\hline 17 & 1600 & 531,180 & 3 th & 50 & 2 & 446th & 513th \\
\hline 18 & 1600 & 511,719 & 3 th & 50 & 2 & 459th & 526 th \\
\hline 19 & 1600 & 460,703 & 3 th & 50 & 2 & 493th & 560th \\
\hline 20 & 2000 & 833,775 & 3th & 40 & 2 & 184th & 234th \\
\hline 21 & 2000 & 802,566 & 3 th & 40 & 2 & 199th & 249th \\
\hline 22 & 2000 & 801,399 & 3 th & 40 & 2 & 200th & 250th \\
\hline 23 & 2000 & 795,657 & 3 th & 40 & 2 & 203th & 253th \\
\hline 24 & 2000 & 785,297 & 3 th & 40 & 2 & 208th & 258th \\
\hline 25 & 2000 & 785,170 & 3 th & 40 & 2 & 208th & 258th \\
\hline
\end{tabular}

Table 4. Initial conditions of all train-sets. 
Table 4. Cont.

\begin{tabular}{|c|c|c|c|c|c|c|c|}
\hline e & $\overline{l_{e}}(\mathrm{~km})$ & $l_{e}^{0}(\mathrm{~km})$ & g & $\mu(\mathrm{d})$ & Interval (d) & $e t a_{e}$ & deadline $_{e}$ \\
\hline 26 & 2000 & 783,766 & 3th & 40 & 2 & 209th & 259th \\
\hline 27 & 2000 & 766,243 & 3 th & 40 & 2 & 217 th & 267 th \\
\hline 28 & 2000 & 757,420 & 5 th & 65 & 1 & 222th & 272th \\
\hline 29 & 2000 & 756,660 & 5 th & 65 & 1 & 222th & 272 th \\
\hline 30 & 2000 & 737,527 & 3 th & 40 & 2 & 232 th & 282th \\
\hline 31 & 2000 & 732,123 & 3 th & 40 & 2 & 234th & 284 th \\
\hline 32 & 2000 & 730,431 & 3 th & 40 & 2 & 235 th & 285 th \\
\hline 33 & 2000 & 721,071 & 3 th & 40 & 2 & 240 th & 290th \\
\hline 34 & 2000 & 720,836 & 3 th & 40 & 2 & 240 th & 290th \\
\hline 35 & 2000 & 718,729 & 3 th & 40 & 2 & 241th & 291th \\
\hline 36 & 2000 & 716,488 & 3 th & 40 & 2 & 242th & 292th \\
\hline 37 & 2000 & 715,068 & 5 th & 65 & 1 & 243th & 293th \\
\hline 38 & 2000 & 688,431 & 5 th & 65 & 1 & 256th & 306th \\
\hline 39 & 2000 & 682,617 & 5 th & 65 & 1 & 259th & 309th \\
\hline 40 & 2000 & 679,690 & 3 th & 40 & 2 & 261th & 311th \\
\hline 41 & 2000 & 679,063 & 3 th & 40 & 2 & 261th & 311th \\
\hline 42 & 2000 & 602,895 & 3 th & 40 & 2 & 299th & 349th \\
\hline 43 & 2000 & 548,553 & 3 th & 40 & 2 & 326 th & 376th \\
\hline 44 & 2000 & 541,090 & 3 th & 40 & 2 & 330th & 380th \\
\hline 45 & 2000 & 539,873 & 3 th & 40 & 2 & 331th & 381th \\
\hline 46 & 2000 & 523,876 & 3 th & 40 & 2 & 339th & 389th \\
\hline 47 & 2000 & 521,226 & 3 th & 40 & 2 & 340 th & 390th \\
\hline 48 & 2000 & 503,254 & 3 th & 40 & 2 & 349th & 399th \\
\hline 49 & 2000 & 501,676 & 3 th & 40 & 2 & 350th & 400th \\
\hline 50 & 2000 & 483,942 & 3 th & 40 & 2 & 359th & 409th \\
\hline 51 & 2000 & 482,739 & 5 th & 65 & 1 & 359th & 409th \\
\hline 52 & 2000 & 460,507 & 5 th & 65 & 1 & 370th & 420th \\
\hline 53 & 2000 & 458,767 & 5 th & 65 & 1 & 371th & 421th \\
\hline 54 & 2000 & 451,664 & 3th & 40 & 2 & 375 th & 425th \\
\hline 55 & 2000 & 311,742 & 3 th & 40 & 2 & 445 th & 495th \\
\hline 56 & 2000 & 305,258 & 3 th & 40 & 2 & 448th & 498th \\
\hline 57 & 2000 & 251,585 & 3 th & 40 & 2 & 475 th & 525 th \\
\hline 58 & 2000 & 242,328 & 3 th & 40 & 2 & 479th & 529 th \\
\hline 59 & 2000 & 205,751 & 3 th & 40 & 2 & 498th & 548 th \\
\hline 60 & 2000 & 182,372 & 3 th & 40 & 2 & 509 th & 559th \\
\hline
\end{tabular}

Based on the data given above, model I and model II were solved, respectively, and both of them obtained the optimal solution. The termination rules for two models were set up with a solver relative gap of 0 in Spyder. One of the detailed results are shown in Table 5, the first column is the identifier (ID) of the train-set; the second column is the start date of HM denoted by $d_{e}$, i.e., the date of delivering train-set to the workshop.

Table 5. Optimal solution.

\begin{tabular}{cccccccccccc}
\hline $\mathbf{e}$ & $\boldsymbol{d}_{\boldsymbol{e}}$ & $\mathbf{e}$ & $\boldsymbol{d}_{\boldsymbol{e}}$ & $\mathbf{e}$ & $\boldsymbol{d}_{\boldsymbol{e}}$ & $\mathbf{e}$ & $\boldsymbol{d}_{\boldsymbol{e}}$ & $\mathbf{e}$ & $\boldsymbol{d}_{\boldsymbol{e}}$ & $\mathbf{e}$ & $\boldsymbol{d}_{\boldsymbol{e}}$ \\
\hline 1 & 108 & 11 & 481 & 21 & 182 & 31 & 280 & 41 & 310 & 51 & 409 \\
2 & 103 & 12 & 142 & 22 & 220 & 32 & 252 & 42 & 349 & 52 & 419 \\
3 & 153 & 13 & 212 & 23 & 250 & 33 & 278 & 43 & 376 & 53 & 421 \\
4 & 193 & 14 & 333 & 24 & 240 & 34 & 273 & 44 & 379 & 54 & 425 \\
5 & 198 & 15 & 360 & 25 & 248 & 35 & 282 & 45 & 381 & 55 & 472 \\
6 & 300 & 16 & 460 & 26 & 188 & 36 & 276 & 46 & 388 & 56 & 498 \\
7 & 312 & 17 & 492 & 27 & 218 & 37 & 238 & 47 & 390 & 57 & 500 \\
8 & 387 & 18 & 458 & 28 & 268 & 38 & 303 & 48 & 398 & 58 & 502 \\
9 & 482 & 19 & 490 & 29 & 270 & 39 & 243 & 49 & 400 & 59 & 496 \\
10 & 482 & 20 & 190 & 30 & 246 & 40 & 308 & 50 & 407 & 60 & 494 \\
\hline
\end{tabular}


To have a macro and a clearer view of the maintenance workload at different periods during the whole year, we presented the number of train-sets be maintained for different periods within the year of 2017 from the optimal solution and the manual plan, and compared them with the predefined number thresholds (see Figure 6).

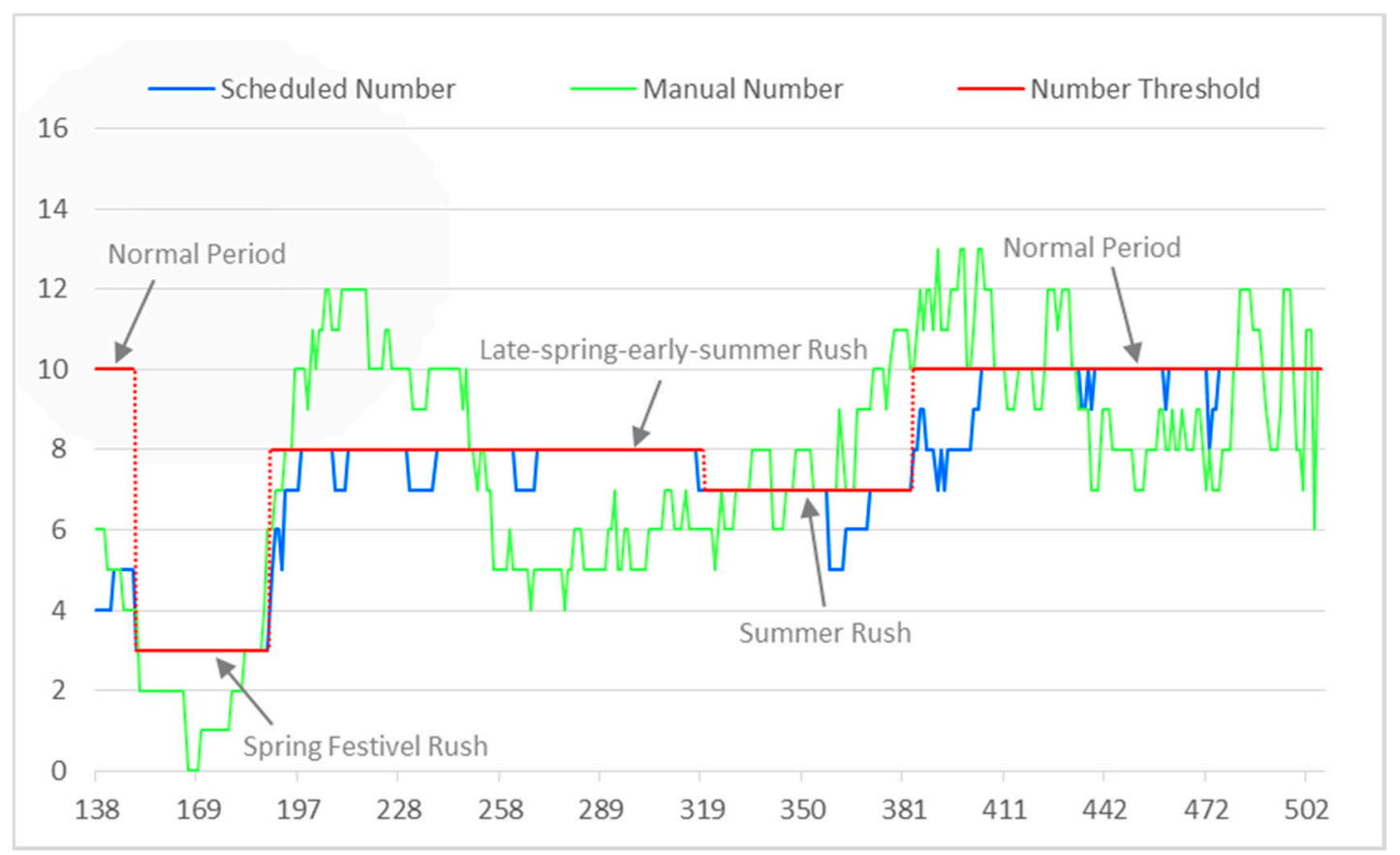

Figure 6. The number of train-sets be maintained for different periods within the year of 2017.

The detailed numerical comparison results of the two models are shown in Table 6. The manual plan is not listed due to it seriously violates the constraints (7), (8), (15) and (16) (see Figure 6).

Table 6. Numerical comparison results.

\begin{tabular}{ccccc}
\hline Model & $\mathbf{z}(\mathbf{k m})$ & Improved Effects & Time Consumption & Improved Effects \\
\hline I & $3,212,769$ & - & $502 \mathrm{~s}$ & - \\
II & $3,212,769$ & 0 & $87 \mathrm{~s}$ & $82.67 \%$ \\
\hline
\end{tabular}

Based on Table 6, we can conclude that our proposed approach is efficiency and effectiveness. A larger $\mathrm{z}$ indicates that more depot facilities and human resources are required. This result also demonstrates that the model II is more efficiency than model I with an improvement of $82.67 \%$ in time consumption. The verification of the resulting HMP by experts demonstrates that the proposed approach is capable of solving large-scale practical problems, and the resulting HMP are reasonable in practice.

\subsection{Sensitivity Analysis}

Sensitivity analysis was performed to evaluate the effects of the variation in three parameters: (1) the average daily operating mileage of train-sets for calculating; (2) HM capacity at the depot; (3) the assumed minimum value of cumulative mileage.

The average daily operating mileage is a key factor determining the deadline for delivering train-sets to the workshop. Because it is an estimated value based on historical data, we evaluate the impact of reducing 100 or increasing 100 in this analysis. 
The available HM capacity limits the train-sets such that not all undergo the HM at the maximum cumulative mileage. This can be adjusted by reallocating human and infrastructure resources. The maintenance capacity includes the maximal number of standard set that can be maintained simultaneously and the receiving capability per day. We analysis the scenarios with 10 standard sets of simultaneously maintenance capacity and two nature sets of receiving capacity at depot per day in this section. The interval of receiving train-sets for depot remains the same.

The purpose of setting the assumed minimum value of cumulative mileage is to control the solution space. If it is too small, there will be no feasible solution existed; if it is too large, much time for solving will be took. Meanwhile, the smaller assumed value, the more date options for delivering train-set to workshop. We set $p=80,90$ and 100 respectively to analysis its sensitivity of calculating time.

According to the variation in the three important parameters, 18 scenarios were created (see as in Table 7). The second, third and fourth column are the amplitude of variation for average daily operating mileage, maintenance capacity (including simultaneously maintenance capacity and receiving capacity) and $p$ respectively. For each scenario, we compare the performance in mileage loss and time consumption under the proposed method. The "+" presents an increase and "-" represents decrease.

Table 7. Sensitivity analysis comparison results.

\begin{tabular}{|c|c|c|c|c|c|c|c|}
\hline Scenario & $l(\mathrm{~km})$ & $B_{2}, N_{2}$ & $p$ & $\mathrm{z}(\mathbf{k m})$ & Improved Effects & $\begin{array}{c}\text { Time } \\
\text { Consumption (s) }\end{array}$ & Improved Effects \\
\hline 1 & 0 & 0,0 & 0 & $3,212,769$ & - & 87 & - \\
\hline 2 & 0 & 0,0 & +10 & $3,212,769$ & 0 & 109 & $-20.18 \%$ \\
\hline 3 & 0 & 0,0 & +20 & $3,177,769$ & $1.09 \%$ & 106 & $-21.84 \%$ \\
\hline 4 & 0 & $+3,+1$ & 0 & $2,394,969$ & $25.45 \%$ & 78 & $10.34 \%$ \\
\hline 5 & 0 & $+3,+1$ & +10 & $2,371,469$ & $26.19 \%$ & 86 & $1.15 \%$ \\
\hline 6 & 0 & $+3,+1$ & +20 & $2,371,469$ & $26.19 \%$ & 98 & $-12.64 \%$ \\
\hline 7 & -100 & 0,0 & 0 & $3,212,769$ & 0 & 81 & $6.90 \%$ \\
\hline 8 & -100 & 0,0 & +10 & $3,212,769$ & 0 & 104 & $-19.54 \%$ \\
\hline 9 & -100 & 0,0 & +20 & $3,177,769$ & $1.09 \%$ & 110 & $-26.44 \%$ \\
\hline 10 & -100 & $+3,+1$ & 0 & $2,394,969$ & $25.45 \%$ & 81 & $6.90 \%$ \\
\hline 11 & -100 & $+3,+1$ & +10 & $2,371,469$ & $26.19 \%$ & 88 & $-1.15 \%$ \\
\hline 12 & -100 & $+3,+1$ & +20 & $2,371,469$ & $26.19 \%$ & 97 & $-11.49 \%$ \\
\hline 13 & +100 & 0,0 & 0 & $3,212,769$ & 0 & 89 & $-2.30 \%$ \\
\hline 14 & +100 & 0,0 & +10 & $3,212,769$ & 0 & 122 & $-40.23 \%$ \\
\hline 15 & +100 & 0,0 & +20 & $3,177,769$ & $1.09 \%$ & 129 & $-48.28 \%$ \\
\hline 16 & +100 & $+3,+1$ & 0 & $2,394,969$ & $25.45 \%$ & 84 & $3.45 \%$ \\
\hline 17 & +100 & $+3,+1$ & +10 & $2,371,469$ & $26.19 \%$ & 88 & $-1.15 \%$ \\
\hline 18 & +100 & $+3,+1$ & +20 & $2,371,469$ & $26.19 \%$ & 100 & $-14.94 \%$ \\
\hline
\end{tabular}

In Table 7, we can see that the variation of average daily operating mileage will has no impact on the optimal objective value, but the result of the HMP will be completely reconstructed; the greater maintenance capability, the less mileage loss and time consumption; variation in the assumed minimum value of cumulative mileage has significant impact on the time consumption. The smaller the assumed value, the longer the time consumption. If the model is infeasible, we can increase the time options to obtain an optimal solution, i.e., increase the value of $p$.

The developed method performed very well in all scenarios. The maximum time consumption (129 s) is from scenario 15, with a greater average daily operating mileage and looser time options. The minimum time consumption (78 s) is from scenario 4 with greater maintenance capacity and tighter time options. However, for any one scenario above, it will take several days to obtain a solution by the manual process. In addition, the manual process is usually infeasible for the whole planning horizon, which needs frequency modification. It can generate a new plan that is efficiently based on our developed method when the condition changes. 


\section{Conclusions}

This research optimizes the electric multiple unit major maintenance plan from the perspective of network design by converting it into the pathway chosen problem on the time-state network. A 0-1 integer linear programming model and its improvements are developed, considering the different transportation demand for passengers and the capacity of the workshop, etc. To control the solution space, a path set generation method is presented. A real-world instance from Shanghai Railway, which is the largest subsidiary in China Railway Corporation, demonstrates that our method can successfully obtain the optimum solution and significantly reduce the solution time to $87 \mathrm{~s}$. Using this decision support tool can help railways with similar characteristics to make the EMU train-set high-level maintenance plan properly.

Future research may investigate the possibility of optimizing the maintenance plan for the first-level and second-level inspections, considering the different transportation demands for passengers and the maintenance capacity of depot under the finite fleet-size of train-sets, and the finite range of the average daily operating mileage of each train-set.

Acknowledgments: This work was supported by the National Natural Science Foundation of China (Grant No. 51378056); the National Railway Administration of the People's Republic of China (Grant No. KF2017-015). We would like to thank Lirong Diao for her valuable comments and suggestions.

Author Contributions: Jianping Wu and Boliang Lin conceived and designed the time-state network, the mathematical model and the path set generation; Jianping $\mathrm{Wu}$ and Jiaxi Wang performed the computes; Jianping $\mathrm{Wu}$, Jiaxi Wang and Siqi Liu analyzed the data and wrote the paper.

Conflicts of Interest: The authors declare no conflict of interest.

\section{References}

1. Stuchly, V.; Grencik, J.; Poprocky, R. Railway vehicle maintenance and information systems. In Proceedings of the International Conference on Computers in Railways VII, Bologna, Italy, 2000; Allan, J., Arias, E., Brebbia, C.A., Eds.; WIT Press: Southampton, UK, 2000; pp. 885-894.

2. Shimada, N. Rolling stock maintenance for safe and stable transport. Jpn. Railw. Eng. 2006, 46, 4-7.

3. Cheng, Y.H.; Tsao, H.L. Rolling stock maintenance strategy selection, spares parts' estimation, and replacements' interval calculation. Int. J. Prod. Econ. 2010, 128, 404-412. [CrossRef]

4. Maróti, G.; Kroon, L. Maintenance routing for train units: The transition model. Transp. Sci. 2005, 39, 518-525. [CrossRef]

5. Maróti, G.; Kroon, L. Maintenance routing for train units: The interchange model. Comput. Oper. Res. 2007, 34, 1121-1140. [CrossRef]

6. Rezvanizaniani1, S.M.; Valibeigloo1, M.; Asghari1, M.; Barabady, J.; Kumar, U. Reliability centered maintenance for rolling stock: A case study in coaches' wheel sets of passenger trains of Iranian railway. In Proceedings of the IEEE International Conference on Industrial Engineering and Engineering Management, Singapore, 8-11 December 2008; Quintana, R., Leung, M., Eds.; IEEE: New York, NY, USA, 2008; pp. 516-520.

7. Tsuji, Y.; Kuroda, M.; Imoto, Y. Rolling stock planning for passenger trains based on ant colony optimization. Trans. Jpn. Soc. Mech. Eng. C 2010, 76, 397-406. [CrossRef]

8. Wang, Y.; Liu, J.; Miao, J.R. Column generation algorithms based optimization method for maintenance scheduling of multiple units. China Railw. Sci. 2010, 31, 115-120.

9. Giacco, G.L.; D'Ariano, A.; Pacciarelli, D. Rolling stock rostering optimization under maintenance constraints. J. Intel. Transp. Syst. 2014, 18, 95-105. [CrossRef]

10. Lai, Y.C.; Fan, D.C.; Huang, K.L. Optimizing rolling stock assignment and maintenance plan for passenger railway operations. Comput. Ind. Eng. 2015, 85, 284-295. [CrossRef]

11. Sriskandarajah, C.; Jardine, A.K.S.; Chan, C.K. Maintenance scheduling of rolling stock using a genetic algorithm. J. Oper. Res. Soc. 1998, 49, 1130-1145. [CrossRef]

12. Lin, B.L.; Lin, R.X. An Approach to the High-level Maintenance Planning for EMU Trains Based on Simulated Annealing. arXiv 2017, arXiv:1704.02752v1. Available online: https:/ / arxiv.org/abs/1704.02752 (accessed on 10 April 2017). 
13. Li, Y.; Zhang, W.J.; Jia, Z.K. Forecast method of annual senior over haul amount for EMU. J. Transp. Eng. 2013, 13, 102-107.

14. Ziaratia, K.; Soumisa, F.; Desrosiers, J.; Gélinas, S.; Saintonge, A. Locomotive assignment with heterogeneous consists at CN North America. Eur. J. Oper. Res. 1997, 97, 281-292. [CrossRef]

15. Lingaya, N.; Cordeau, J.F.; Desaulniers, G.; Desrosiers, J.; Soumis, F. Operational car assignment at VIA Rail Canada. Transp. Res. B Methodol. 2002, 36, 755-778. [CrossRef]

16. Wang, L.; Ma, J.J.; Lin, B.L.; Chen, L.; Wen, X.H. Method for optimization of freight locomotive scheduling and routing problem. J. China Railw. Soc. 2014, 36, 7-15.

17. Moudania, W.E.; Félix, M.C. A dynamic approach for aircraft assignment and maintenance scheduling by airlines. J. Air Transp. Manag. 2000, 6, 233-237. [CrossRef]

18. Budai, G.; Huisman, D.; Dekker, R. Scheduling preventive railway maintenance activities. J. Oper. Res. Soc. 2006, 57, 1035-1044. [CrossRef]

19. Mehmet, B.; Bilge, Ü. Operational aircraft maintenance routing problem with remaining time consideration. Eur. J. Oper. Res. 2014, 235, 315-328.

20. Grigoriev, A.; Klundert, J.V.D.; Spieksma, F.C.R. Modeling and solving the periodic maintenance problem. Eur. J. Oper. Res. 2006, 172, 783-797. [CrossRef]

21. Lv, Y.; Zhang, J.; Qin, W. A genetic regulatory network-based method for dynamic hybrid flow shop scheduling with uncertain processing times. Appl. Sci. 2017, 7, 23. [CrossRef]

22. Shan, R.; Zhao, Z.S.; Chen, P.F.; Liu, W.J.; Xiao, S.Y.; Hou, Y.H.; Wang, Z. Network modeling and assessment of ecosystem health by a multi-population swarm optimized neural network ensemble. Appl. Sci. 2016, 6, 175. [CrossRef]

23. China Railway. User Manual for EMU Operation and Maintenance Procedures, 1st ed.; Railway Publishing House: Beijing, China, 2013.

(C) 2017 by the authors. Licensee MDPI, Basel, Switzerland. This article is an open access article distributed under the terms and conditions of the Creative Commons Attribution (CC BY) license (http:/ / creativecommons.org/licenses/by/4.0/). 